\title{
Comparative effects of valsartan in combination with cilnidipine or amlodipine on cardiac remodeling and diastolic dysfunction in Dahl salt-sensitive rats
}

\author{
Kai Nagasawa ${ }^{1}$, Keiji Takahashi ${ }^{1}$, Natsumi Matsuura ${ }^{1}$, Miwa Takatsu$^{1}$, Takuya Hattori ${ }^{1}$, Shogo Watanabe ${ }^{1}$, \\ Eri Harada ${ }^{2}$, Kazumi Niinuma ${ }^{2}$, Toyoaki Murohara ${ }^{3}$ and Kohzo Nagata ${ }^{1}$
}

Angiotensin receptor blockers (ARBs) are often supplemented with calcium channel blockers (CCBs) for treatment of hypertension. We recently showed that the L/N-type CCB cilnidipine has superior cardioprotective effects compared with the L-type CCB amlodipine in Dahl salt-sensitive (DS) rats. We have now compared the effects of the ARB valsartan combined with cilnidipine or amlodipine on cardiac pathophysiology in DS rats. DS rats fed a high-salt diet from 6 weeks of age were treated with vehicle, valsartan alone $\left(10 \mathrm{mg} \mathrm{kg}^{-1}\right.$ per day), or valsartan combined with either cilnidipine $\left(1 \mathrm{mg} \mathrm{kg}^{-1}\right.$ per day) or amlodipine ( $1 \mathrm{mg} \mathrm{kg}^{-1}$ per day) from 7 to 11 weeks. The salt-induced increase in systolic blood pressure apparent in the vehicle group was attenuated similarly in the three drug treatment groups. Valsartan-cilnidipine attenuated left ventricular (LV) fibrosis and diastolic dysfunction as well as cardiac oxidative stress and inflammation to a greater extent than did valsartan alone or valsartan-amlodipine. In addition, the increases in urinary excretion of dopamine and epinephrine as well as in cardiac renin-angiotensin-aldosterone-system (RAAS) gene expression apparent in vehicle-treated rats were attenuated to a greater extent by valsartan-cilnidipine than by the other two treatments. Valsartan-cilnidipine thus attenuated LV remodeling and diastolic dysfunction more effectively than did valsartan or valsartan-amlodipine in rats with salt-sensitive hypertension, and this superior cardioprotective action of valsartan-cilnidipine compared with valsartan-amlodipine is likely attributable, at least in part, to the greater antioxidant and antiinflammatory effects associated with both greater inhibition of cardiac RAAS gene expression and $\mathrm{N}$-type calcium channel blockade.

Hypertension Research (2015) 38, 39-47; doi:10.1038/hr.2014.136; published online 11 September 2014

Keywords: angiotensin receptor blocker; calcium channel blocker; cardiac remodeling; diastolic function

\section{INTRODUCTION}

Hypertension is a major risk factor for cardiovascular disease and the most common risk factor for death from any cause. ${ }^{1,2}$ For hypertensive patients who do not respond to monotherapy, combinations of two or more antihypertensive drugs are recommended. ${ }^{3}$ Combination therapies with an angiotensin receptor blocker (ARB) and either a thiazide diuretic or a calcium channel blocker (CCB), or both, are commonly administered to achieve strict blood pressure control and to prevent target organ damage. ${ }^{4}$ However, the organ-protective action has been found to vary depending on CCB type. ${ }^{5}$ Amlodipine is a dihydropyridine-based CCB and blocks L-type calcium channels that are expressed predominantly in vessels and regulate vascular tone. Cilnidipine is also a dihydropyridine-based CCB but blocks both Nand L-type calcium channels. ${ }^{6,7} \mathrm{~N}$-type calcium channels are expressed in, and are implicated in the release of norepinephrine from, sympathetic nerve terminals. ${ }^{8}$
Several actions of cilnidipine, including suppression of sympathetic nerve activity, 9,10 renal protection ${ }^{11,12}$ and cardiovascular protection, ${ }^{13,14}$ are thought to be mediated by its inhibitory effect on N-type calcium channels. We recently showed that cilnidipine ameliorated left ventricular (LV) concentricity, fibrosis and diastolic dysfunction to a greater extent than did amlodipine in rats with salt-sensitive hypertension. ${ }^{15}$ The Cilnidipine vs. Amlodipine Randomised Trial for Evaluation in Renal Disease study also revealed that cilnidipine is superior to amlodipine in preventing the progression of proteinuria in hypertensive patients also treated with an inhibitor of the renin-angiotensin-aldosterone system (RAAS). ${ }^{16}$ The mechanism underlying the superior cardioprotective action of an N/L-type CCB added to an ARB has remained unclear, however.

We have now investigated the effects of the combination of cilnidipine and the ARB valsartan, in comparison with amlodipine

${ }^{1}$ Department of Pathophysiological Laboratory Sciences, Nagoya University Graduate School of Medicine, Nagoya, Japan; ${ }^{2}$ Pharmacology Research Laboratory, Research Institute, Ajinomoto Pharmaceuticals Co., Ltd., Kawasaki, Japan and ${ }^{3}$ Department of Cardiology, Nagoya University Graduate School of Medicine, Nagoya, Japan

Correspondence: Professor K Nagata, Department of Pathophysiological Laboratory Sciences, Nagoya University Graduate School of Medicine, 1-1-20 Daikominami, Higashi-ku, Nagoya 461-8673, Japan.

E-mail: nagata@met.nagoya-u.ac.jp

Received 19 March 2014; revised 30 July 2014; accepted 12 August 2014; published online 11 September 2014 
plus valsartan as well as with valsartan alone, on cardiac pathophysiology in rats with salt-sensitive hypertension.

\section{MATERIALS AND METHODS}

\section{Animals and experimental protocols}

Five-week-old male inbred Dahl salt-sensitive (DS) rats were obtained from Japan SLC (Hamamatsu, Japan), were handled in accordance with the guidelines of Nagoya University Graduate School of Medicine as well as with the Guide for the Care and Use of Laboratory Animals (NIH publication no. $85-23$, revised 1996), and were fed laboratory chow containing $0.36 \% \mathrm{NaCl}$ until 6 weeks of age. Rats fed an $8 \% \mathrm{NaCl}$ diet after 6 weeks of age were treated with valsartan alone $\left(10 \mathrm{mg} \mathrm{kg}^{-1}\right.$; Livzon Group Changzhou Kony Pharmaceutical, Changzhou, China) (Val group, $n=11)$, valsartan $\left(10 \mathrm{mg} \mathrm{kg}^{-1}\right)$ plus amlodipine ( $1 \mathrm{mg} \mathrm{kg}^{-1}$; Lek, Kolodvorska, Slovenia) (VAml group, $n=11$ ), valsartan $\left(10 \mathrm{mg} \mathrm{kg}^{-1}\right)$ plus cilnidipine $\left(1 \mathrm{mg} \mathrm{kg}^{-1}\right.$; Ajinomoto, Tokyo, Japan) (VCil group, $n=11$ ) or vehicle (LVH group, $n=6$ ) from 7 weeks of age. The doses of valsartan, amlodipine and cilnidipine were determined on the basis of the results of a previous study. ${ }^{17}$ The rats treated with vehicle gradually developed pronounced hypertension and manifested hypertension-induced compensated LV hypertrophy (LVH) at 11 weeks of age. DS rats maintained on a $0.36 \% \mathrm{NaCl}$ diet after 6 weeks of age remain normotensive, and such animals served as age-matched controls (CONT group, $n=6$ ). Each diet and tap water were provided ad libitum throughout the experimental period. At 11 weeks of age, all rats were anesthetized by intraperitoneal injection of ketamine $\left(50 \mathrm{mg} \mathrm{kg}^{-1}\right)$ and xylazine $\left(10 \mathrm{mg} \mathrm{kg}^{-1}\right)$ and were subjected to echocardiographic and hemodynamic analyses. The animals were then killed, the heart was excised and LV tissue was separated for analysis.

\section{Blood pressure measurement, echocardiography and hemodynamics}

Systolic blood pressure (SBP) was measured weekly in conscious animals by tail-cuff plethysmography (BP-98A; Softron, Tokyo, Japan). At 11 weeks of age, rats were subjected to transthoracic echocardiography as previously described. ${ }^{15}$ In brief, M-mode echocardiography was performed with a $12.5-\mathrm{MHz}$ transducer (Xario SSA-660A; Toshiba Medical Systems, Tochigi, Japan). In addition, the pulsed Doppler echocardiographic data were analyzed for assessment of LV diastolic function. ${ }^{15}$ After echocardiography, a $2 \mathrm{~F}$ micromanometer-tipped catheter (SPR-320; Millar Instruments, Houston, TX, USA) that had been calibrated relative to atmospheric pressure was inserted through the right carotid artery into the left ventricle. ${ }^{18}$ Tracings of LV pressure and the electrocardiogram were digitized to determine LV end-diastolic pressure (LVEDP). The time constant of isovolumic relaxation (tau) was calculated by the derivative method of Raff and Glantz, as described previously. ${ }^{19}$

\section{Histology and immunohistochemistry}

LV tissue was fixed with ice-cold $4 \%$ paraformaldehyde for $48 \mathrm{~h}$, embedded in paraffin, and processed for histology as described previously. ${ }^{15}$ Transverse sections were stained either with hematoxylin-eosin for routine histological examination or with Azan-Mallory solution for evaluation of fibrosis. To evaluate macrophage infiltration into the myocardium, we performed immunostaining for the monocyte-macrophage marker CD68 with the paraffinembedded sections. ${ }^{20}$ All image analysis was performed with NIH Scion Image software (Scion, Frederick, MD, USA).

\section{Measurement of biochemical parameters}

A blood sample was collected from the right carotid artery of rats deprived of food overnight under anesthesia by intraperitoneal injection of sodium pentobarbital $\left(50 \mathrm{mg} \mathrm{kg}^{-1}\right)$ and was centrifuged at $1400 \mathrm{~g}$ for $10 \mathrm{~min}$ at room temperature. The resultant serum or plasma supernatants were stored at $-80^{\circ}$ $\mathrm{C}$ until analysis. Plasma renin activity was determined by radioimmunoassay with the use of renin RIA beads (Abbott Japan, Tokyo, Japan). Plasma angiotensin II concentration was determined by radioimmunoassay as described previously, ${ }^{21}$ and serum aldosterone concentration was measured by radioimmunoassay with the use of a DPC aldosterone kit (Mitsubishi Chemical Medience, Tokyo, Japan). Urinary catecholamine concentrations were measured by high-performance liquid chromatography, and urinary catecholamine excretion over $24 \mathrm{~h}$ was calculated as described previously. ${ }^{22}$

\section{Assay of superoxide production}

Reduced nicotinamide adenine dinucleotide phosphate (NADPH)-dependent superoxide production by homogenates of freshly frozen LV tissue was measured with an assay based on lucigenin-enhanced chemiluminescence as described previously. ${ }^{15}$ The average of dihydroethidium fluorescence intensity values was calculated with the use of NIH Image software (ImageJ). ${ }^{23}$

\section{Quantitative RT-PCR analysis}

Total RNA was extracted from LV tissue and treated with deoxyribonuclease with the use of a spin-vacuum total RNA isolation kit (Promega, Madison, WI, USA). Portions of the RNA ( $2 \mu \mathrm{g})$ were subjected to reverse transcription (RT) with the use of a PrimerScript RT Reagent Kit (Takara, Shiga, Japan). Quantitative PCR analysis was performed with the use of SYBR Mix Ex Taq II (Takara), a Thermal Cycler Dice Real Time System II (Takara) and specific primers for cDNAs encoding atrial natriuretic peptide, ${ }^{20}$ brain natriuretic peptide, ${ }^{20}$ collagen type I or III, ${ }^{20}$ transforming growth factor (TGF)- $\beta 1,{ }^{20}$ connective tissue growth factor, ${ }^{20}$ monocyte chemoattractant protein (MCP)$1,{ }^{20}$ osteopontin, ${ }^{15}$ angiotensin-converting enzyme, ${ }^{20}$ the $\mathrm{AT}_{1 \mathrm{~A}}$ receptor (type $1 \mathrm{~A}$ receptor for angiotensin II) ${ }^{20}$ the mineralocorticoid receptor, ${ }^{20}$ serum/ glucocorticoid-regulated kinase 1 (Sgk1), ${ }^{20}$ or the $\mathrm{p} 22^{\text {phox }}$ or gp9 $91^{\text {phox }}$ subunits of NADPH oxidase. ${ }^{20}$ Reagents for detection of human glyceraldehyde-3phosphate dehydrogenase (GAPDH) mRNA (Applied Biosystems, Foster City, CA, USA) were used to quantify rat GAPDH mRNA as an internal standard.

\section{Statistical analysis}

Data are presented as means \pm s.e.m. Differences among groups of rats at 11 weeks of age were assessed by one-way factorial analysis of variance; if a significant difference was detected, intergroup comparisons were performed with Fisher's multiple-comparison test. The time course of SBP was compared among groups by two-way repeated-measures analysis of variance. A $P$-value of $<0.05$ was considered as statistically significant.

\section{RESULTS}

\section{LV geometry and function}

SBP was similar in all groups of rats at 6 weeks of age, but it was significantly higher in the LVH group than in the CONT group at 7 weeks of age and thereafter (Figure 1; Table 1). At 9 weeks and thereafter, SBP was significantly reduced in the Val, VAml and VCil groups compared with the LVH group. Although the overall time course of SBP did not differ among the three treatment groups, SBP was significantly lower in the VAml and VCil groups than in the Val group at 11 weeks. The ratios of heart or LV weight to tibial length, indices of cardiac and $\mathrm{LVH}$, respectively, were significantly increased in the LVH group compared with the CONT group at 11 weeks, and these effects were attenuated in all treatment groups (Table 1).

Echocardiography revealed that both LV end-diastolic dimension (LVDd) and LV end-systolic dimension were decreased, and that interventricular septum thickness, LV posterior wall thickness, LV fractional shortening, LV ejection fraction, LV mass and relative wall thickness (RWT) were increased in the LVH group compared with the CONT group (Table 2). All of these changes were attenuated in all treatment groups, and that in RWT was inhibited to a greater extent in the VCil group than in the Val and VAml groups. The isovolumic relaxation time, deceleration time and tau, all of which are indices of LV relaxation, as well as the ratio of LVEDP to LVDd, an index of LV diastolic stiffness, were increased in the LVH group compared with the CONT group. All of these effects were attenuated to a greater extent in the VCil group than in the Val or VAml groups. 


\section{Cardiomyocyte hypertrophy and cardiac fibrosis}

The cross-sectional area of LV cardiomyocytes was increased in the LVH group compared with the CONT group, and this increase was attenuated to a greater extent in the VAml and VCil groups than in the Val group (Figures 2a and b). Hemodynamic overload also resulted in marked upregulation of atrial natriuretic peptide and brain natriuretic peptide gene expression in the left ventricle, and the increased expression of these fetal-type cardiac genes was similarly attenuated in both VAml and VCil groups (Figures $2 \mathrm{c}$ and d).

Azan-Mallory staining revealed that fibrosis in the perivascular and interstitial regions of the LV myocardium was increased in the LVH group compared with the CONT group, and these increases were partially attenuated in the Val and VAml groups and completely inhibited in the VCil group (Figures $3 \mathrm{a}-\mathrm{c}$ ). The abundance of collagen types I and III mRNAs was increased in the left ventricle of rats in the LVH group, and these effects were attenuated to a greater extent in the VCil group than in the Val or VAml groups (Figures $3 \mathrm{~d}$ and e). The amounts of TGF- $\beta 1$ and connective tissue growth factor mRNAs in the left ventricle were also significantly increased in the LVH group compared with the CONT group, and these effects were attenuated in the VAml group and to a greater extent in the VCil group (Figures $3 \mathrm{f}$ and $\mathrm{g}$ ).

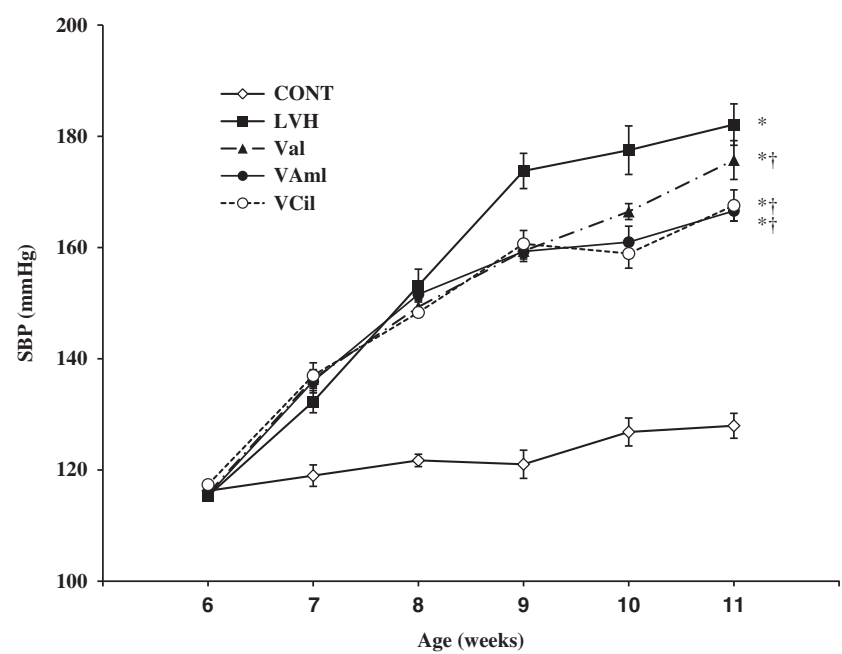

Figure 1 Time course of SBP in rats of the five experimental groups. Data are means \pm s.e.m. $(n=6,6,11,11$ and 11 rats for the CONT, LVH, Val, VAml and VCil groups, respectively). ${ }^{*} P<0.05$ vs. CONT group; ${ }^{\dagger} P<0.05$ vs. LVH group.

\section{Cardiac oxidative stress}

Both superoxide production in myocardial tissue sections as revealed by staining with dihydroethidium as well as the activity of NADPH oxidase in LV homogenates were markedly increased in the LVH group compared with the CONT group, and these effects were inhibited to a greater extent in the VCil group than in the Val and VAml groups (Figures $4 \mathrm{a}-\mathrm{c}$ ). The expression of genes for the $\mathrm{p} 22^{\mathrm{phox}}$ and gp91 ${ }^{\text {phox }}$ membrane components of NADPH oxidase was also upregulated in the left ventricle of rats in the LVH group, and these effects were inhibited to a greater extent in the VCil group than in the Val or VAml groups (Figures $4 \mathrm{~d}$ and e).

\section{Cardiac inflammation}

Immunostaining for the monocyte-macrophage marker CD68 revealed that macrophage infiltration in the LV myocardium was increased in the LVH group compared with the CONT group and that this effect of salt loading was attenuated to a greater extent in the VCil group than in the Val and VAml groups (Figures $5 \mathrm{a}$ and $\mathrm{b}$ ). The expression of MCP-1 and osteopontin genes in the left ventricle was also increased in the LVH group, and these effects were inhibited to a greater extent in the VCil group than in the Val or VAml groups (Figures $5 \mathrm{c}$ and $\mathrm{d}$ ).

\section{RAAS and sympathetic activity}

Salt loading reduced plasma renin activity as well as plasma angiotensin II and serum aldosterone concentrations, and these effects were not affected by any of the antihypertensive drugs (Table 1). Expression of the angiotensin-converting enzyme gene was significantly upregulated in the left ventricle of rats in the LVH group compared with the CONT group, and this effect was inhibited to a greater extent in the VCil group than in the Val and VAml groups (Figure 6a). Expression of the $\mathrm{AT}_{1 \mathrm{~A}}$ receptor gene was similar in CONT and LVH groups and was inhibited to a greater extent in the VCil group than in the Val and VAml groups (Figure $6 \mathrm{~b}$ ). The abundance of mineralocorticoid receptor mRNA was significantly increased in the LVH group compared with the CONT group, and this effect was attenuated in the Val and VAml groups and to a greater extent in the VCil group (Figure 6c). The upregulation of Sgk1 gene expression apparent in the LVH group was attenuated in the VCil group but not in the Val or VAml groups (Figure $6 \mathrm{~d}$ ).

Urinary excretion of norepinephrine, dopamine and epinephrine was markedly increased in the LVH group compared with the CONT group (Figures $6 \mathrm{e}-\mathrm{g}$ ). Whereas the increase in urinary excretion of norepinephrine was similarly attenuated in all treatment groups, given

Table 1 Anatomic, metabolic and hormonal parameters in rats of the five experimental groups at 11 weeks of age

\begin{tabular}{|c|c|c|c|c|c|}
\hline Parameter & CONT & $\angle V H$ & Val & $V A m I$ & VCil \\
\hline Body weight (g) & $344.7 \pm 4.9$ & $342.0 \pm 7.5$ & $338.6 \pm 6.3$ & $325.9 \pm 3.8^{a}$ & $333.7 \pm 3.9$ \\
\hline $\mathrm{SBP}(\mathrm{mm} \mathrm{Hg})$ & $128.0 \pm 1.7$ & $182.1 \pm 3.6^{\mathrm{a}}$ & $175.7 \pm 2.5^{\mathrm{a}}$ & $166.6 \pm 3.0^{\mathrm{a}, \mathrm{b}, \mathrm{c}}$ & $167.6 \pm 1.7^{a, b, c}$ \\
\hline Heart weight/tibial length $\left(\mathrm{mg} \mathrm{mm}^{-1}\right)$ & $27.73 \pm 0.75$ & $38.14 \pm 0.63^{a}$ & $33.31 \pm 0.95^{\mathrm{a}, \mathrm{b}}$ & $32.08 \pm 0.62^{a, b}$ & $32.37 \pm 0.76^{a, b}$ \\
\hline LV weight/tibial length $\left(\mathrm{mg} \mathrm{mm}^{-1}\right)$ & $20.76 \pm 0.58$ & $28.69 \pm 0.50^{a}$ & $25.51 \pm 0.56^{a, b}$ & $24.61 \pm 0.45^{a, b}$ & $24.78 \pm 0.55^{a, b}$ \\
\hline Plasma renin activity $\left(\mathrm{ng} \mathrm{ml}^{-1} \mathrm{~h}^{-1}\right)$ & $5.76 \pm 0.38$ & $0.30 \pm 0.18^{a}$ & $0.52 \pm 0.12^{\mathrm{a}}$ & $0.52 \pm 0.13^{\mathrm{a}}$ & $0.46 \pm 0.12^{\mathrm{a}}$ \\
\hline
\end{tabular}

Abbreviations: LV, left ventricular; LVH, LV hypertrophy; SBP, systolic blood pressure. Data are means \pm s.e.m. ( $n=6,6,11,11$ and 11 rats for the CONT, LVH, Val, VAml and VCil groups, respectively.

a $P<0.05$ vs. CONT group.

b $P<0.05$ vs. CONT group.
vVH group.

${ }^{\mathrm{b}} P<0.05$ vs. LVH group.
${ }^{\mathrm{b}}<0.05$ vs. Val group. 
Table 2 Cardiac morphological and functional parameters in rats of the five experimental groups at 11 weeks of age

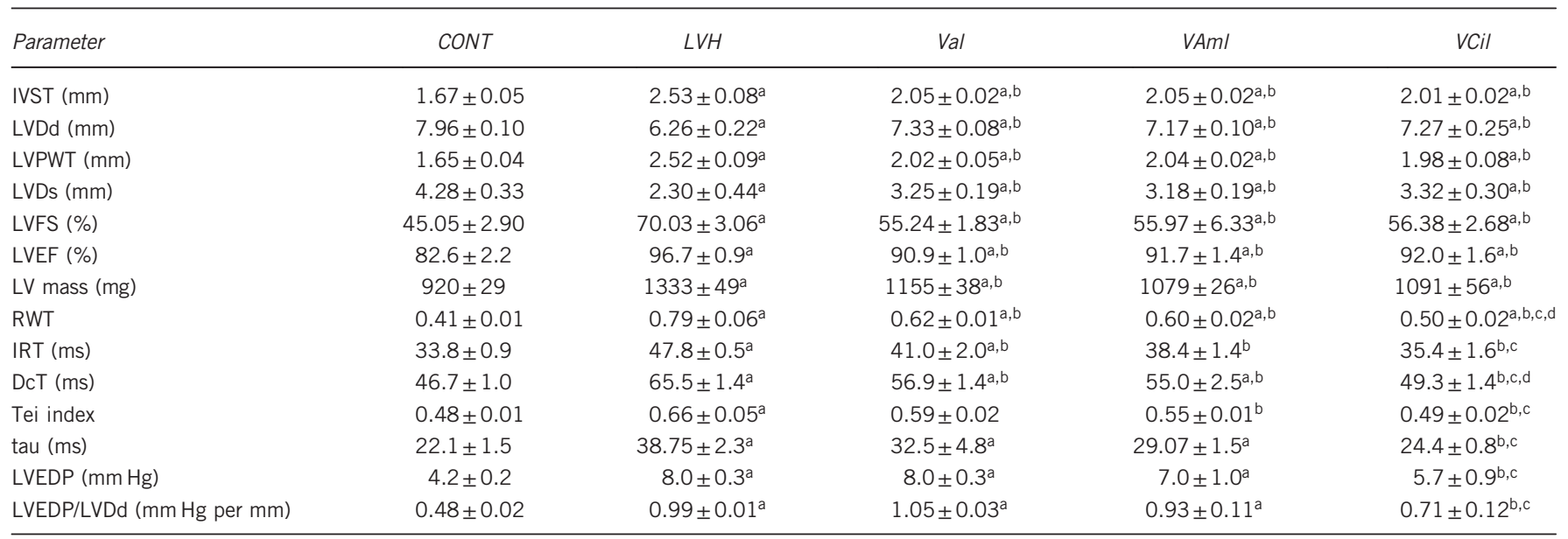

Abbreviations: DcT, deceleration time; IVST, interventricular septum thickness; LV, left ventricular; LVDd, LV end-diastolic dimension; LVDs, LV end-systolic dimension; LVEDP, LV end-diastolic pressure; LVFS, LV fractional shortening; LVEF, LV ejection fraction; LVPWT, LV posterior wall thickness; IRT, isovolumic relaxation time; RWT, relative wall thickness; tau, time constant of isovolumic relaxation. Data are means \pm s.e.m. $(n=6,6,11,11$ and 11 for the CONT, LVH, Val, VAml and VCil groups, respectively.

a $P<0.05$ vs. CONT group.

${ }^{\mathrm{b}} P<0.05$ vs. LVH group.

${ }^{c} P<0.05$ vs. Val group.

d $P<0.05$ vs. VAml group

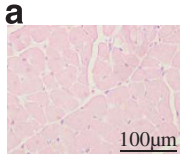

CONT

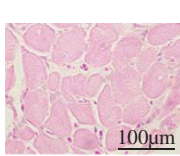

LVH

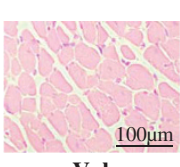

Val

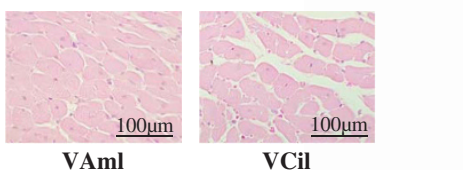

VAml VCil

C

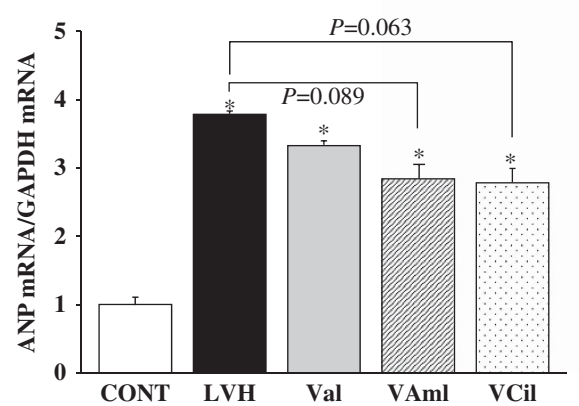

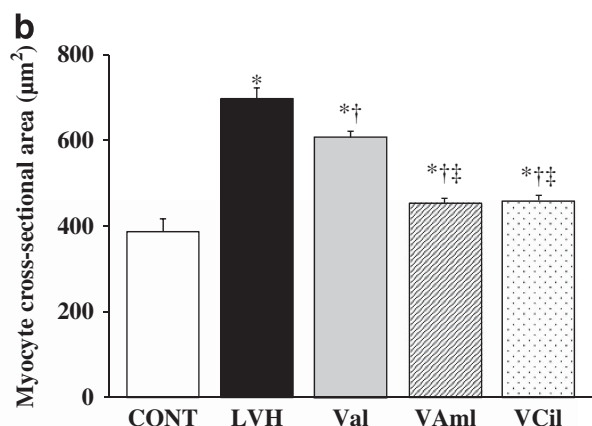

d

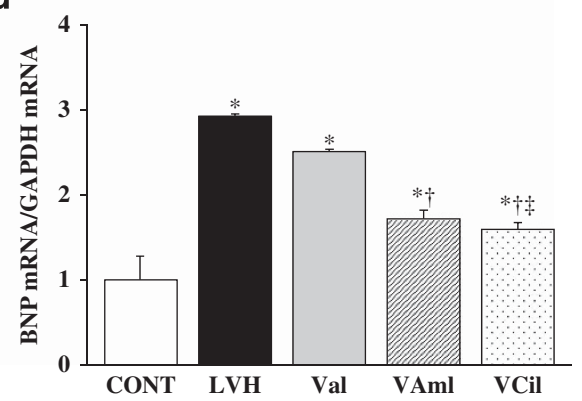

Figure 2 Myocyte cross-sectional area and expression of fetal-type cardiac genes in the left ventricle of rats in the five experimental groups at 11 weeks of age. (a) Hematoxylin-eosin staining of transverse sections of the LV myocardium. Scale bars, $100 \mu \mathrm{m}$. (b) Cross-sectional area of cardiac myocytes determined from sections similar to those in (a). (c, d) Quantitative RT-PCR analysis of atrial natriuretic peptide (ANP) and brain natriuretic peptide (BNP) mRNAs, respectively. The amount of each mRNA was normalized by that of GAPDH mRNA and then expressed relative to the mean value for the CONT group. Data in (b) through (d) are means \pm s.e.m. $\left(n=6,6,11,11\right.$ and 11 rats for the CONT, LVH, Val, VAml and VCil groups, respectively). ${ }^{*}<0.05$ vs. CONT group; ${ }^{\dagger} P<0.05$ vs. LVH group; ${ }^{\ddagger} P<0.05$ vs. Val group.

that in dopamine or epinephrine excretion was attenuated to a greater extent in the VCil group than in the Val and VAml groups.

\section{DISCUSSION}

We have shown here that valsartan monotherapy as well as combination therapy with valsartan and either amlodipine or cilnidipine similarly attenuated hypertension and LVH in DS hypertensive rats, whereas the combination of valsartan and cilnidipine ameliorated LV concentricity, fibrosis and diastolic dysfunction to a greater extent than did valsartan alone or the combination of valsartan and amlodipine. The superior cardioprotective effects of the combination of valsartan and cilnidipine were associated with more effective inhibition of cardiac oxidative stress, inflammation and RAAS gene expression. The sympatholytic action of cilnidipine, as revealed by its 
a
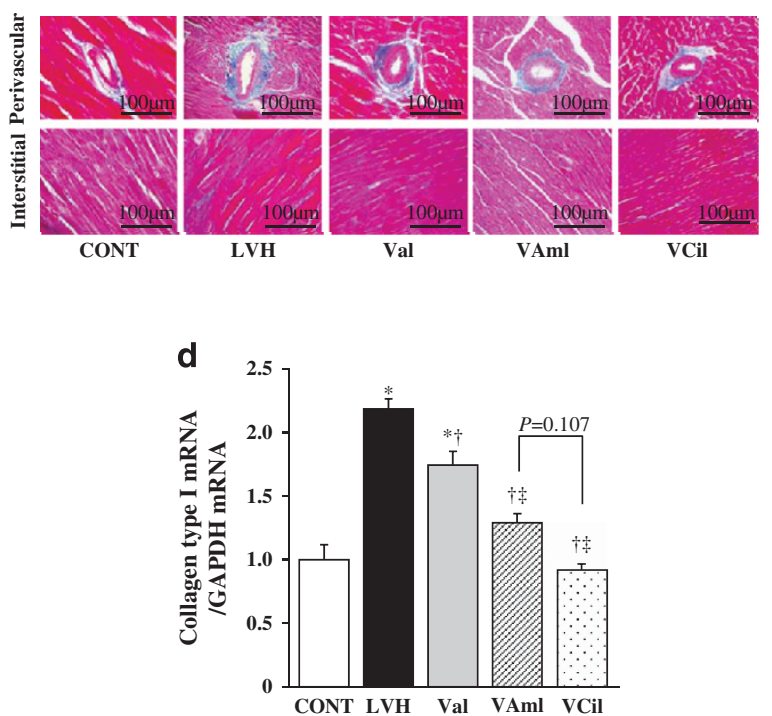

f

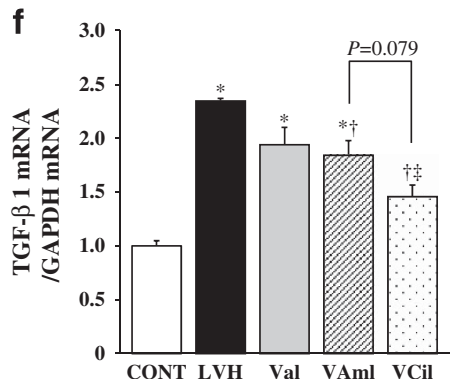

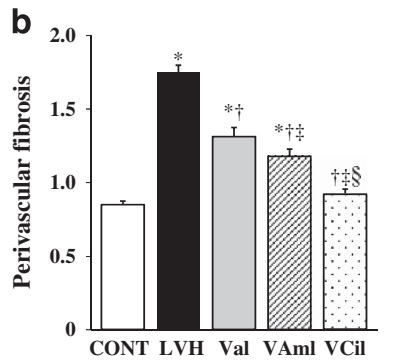

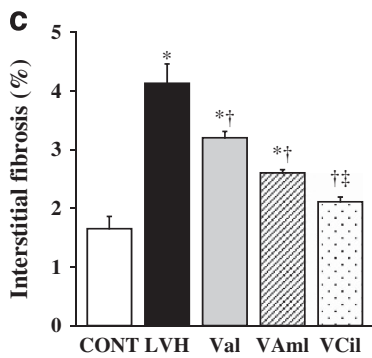

e

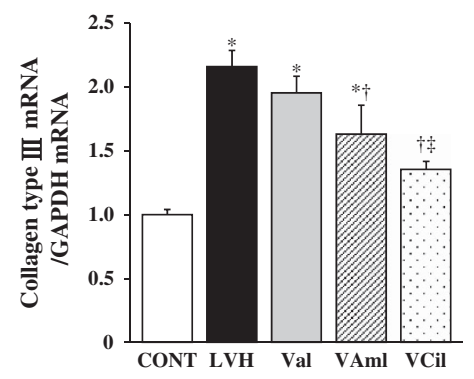

g

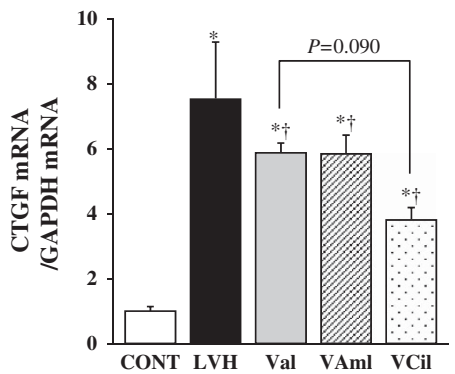

Figure 3 Cardiac fibrosis in the left ventricle of rats in the five experimental groups at 11 weeks of age. (a) Collagen deposition as revealed by Azan-Mallory staining in perivascular or interstitial regions of the myocardium. Scale bars, $100 \mu \mathrm{m}$. (b, c) Relative extents of perivascular and interstitial fibrosis, respectively, in the LV myocardium as determined from sections similar to those in (a). (d-g) Quantitative RT-PCR analysis of collagen type I, collagen type III, TGF- $\beta 1$ and connective tissue growth factor (CTGF) mRNAs, respectively. The abundance of each mRNA was normalized by that of GAPDH mRNA and then expressed relative to the mean value for the CONT group. Data in (b) through (g) are means \pm s.e.m. $(n=6,6,11,11$ and 11 rats for the CONT, LVH, Val, VAml and VCil groups, respectively). ${ }^{*} P<0.05$ vs. CONT group; ${ }^{\dagger} P<0.05$ vs. LVH group; ${ }^{\ddagger} P<0.05$ vs. Val group; ${ }^{\S} P<0.05$ vs. VAml group.

effect on urinary catecholamine (dopamine and epinephrine) excretion, might also have contributed to the more effective amelioration of cardiac remodeling and diastolic dysfunction by the combination of valsartan and cilnidipine in comparison with the other drug treatment groups.

All of the treatment groups manifested attenuation of cardiac hypertrophy, which did not differ significantly between the Val group and the combination therapy groups. This latter finding might be due to the fact that the combination therapies reduced SBP to a greater extent than did valsartan alone only at 11 weeks of age. In a previous study of the same animal model with equivalent doses of drugs and similar experimental protocols, both the Val group and the combination therapy groups manifested significant attenuation of the increase in SBP within 1 week of treatment onset. ${ }^{17}$ Although the reason for this discrepancy remains unclear, it is possible that differences in the time to measure blood pressure or breeding conditions may have affected the time course of SBP.

Sympathetic hyperactivity results in activation of the RAAS ${ }^{24,25}$ and is considered as a hallmark of hypertensive cardiovascular disease morbidity and mortality, ${ }^{26}$ given that chronic activation of the sympathetic nervous system has been shown to have adverse effects on the myocardium and peripheral circulation. These effects are thought to contribute to cardiac and vascular structural alterations that may advance disease progression. ${ }^{27}$ Many studies have shown that cilnidipine suppresses sympathetic nerve activity and the RAAS, ${ }^{28,29}$ likely as a result of its inhibition of N-type calcium channels. In the present study, urinary norepinephrine excretion was suppressed to a similar extent in all three treatment groups. However, the increases in the urinary excretion of dopamine and epinephrine apparent in the LVH group were suppressed to a greater extent in the VCil group than in the Val and VAml groups, suggesting that valsartan suppressed sympathetic hyperactivity but that cilnidipine also contributed to such suppression. The increase in RWT was attenuated to a greater extent in the VCil group than in the Val and VAml groups. Furthermore, although the suppression of the systemic RAAS by salt loading was not affected by any of the antihypertensive drug treatments, the expression levels of RAAS-related genes in the heart tended to be lower in the VCil group than in the Val and VAml groups, suggesting that the cardiac RAAS may have been inhibited to a greater extent by the combination of valsartan and cilnidipine. It is thus possible that LVH per se is primarily load dependent, ${ }^{30}$ whereas LV concentricity is affected by cardiac sympathetic activity and the RAAS as well as by load. ${ }^{31}$ 
a

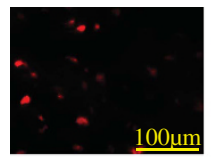

CONT

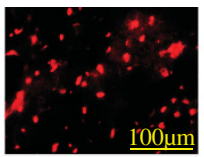
LVH

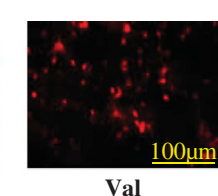

Val

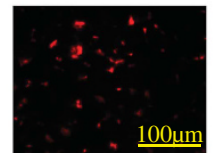

VAml

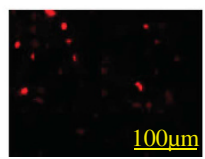

VCil b

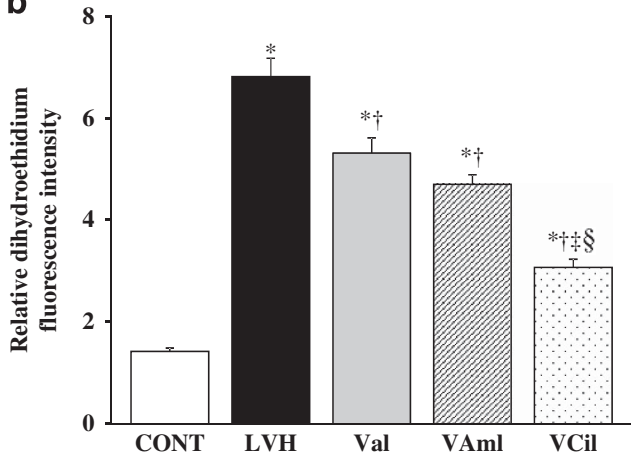

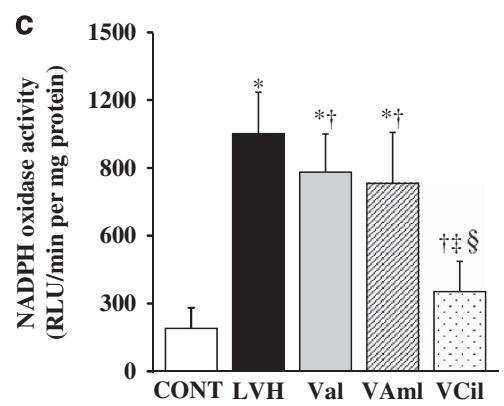
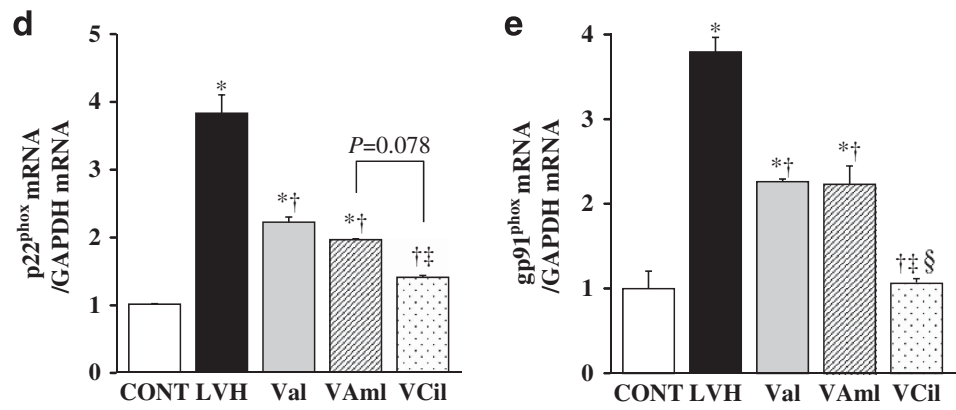

Figure 4 Superoxide production as well as NADPH oxidase activity and gene expression in the left ventricle of rats in the five experimental groups at 11 weeks of age. (a) Superoxide production as revealed by dihydroethidium staining in sections of the LV myocardium. Scale bars, $100 \mu \mathrm{m}$. (b) Quantitation of dihydroethidium fluorescence intensity in sections similar to those in (a). (c) NADPH-dependent superoxide production in LV homogenates. Results are expressed as relative light units (RLUs) per minute per milligram of protein. (d, e) Quantitative RT-PCR analysis of p22 ${ }^{\text {phox }}$ and gp91 ${ }^{\text {phox }}$ mRNAs, respectively. The amount of each mRNA was normalized by that of GAPDH mRNA and then expressed relative to the mean value for the CONT group. Data in (b) through (e) are means \pm s.e.m. $(n=6,6,11,11$ and 11 rats for the CONT, LVH, Val, VAml and VCil groups, respectively). * $P<0.05$ vs. CONT group; ${ }^{\dagger} P<0.05$ vs. LVH group; ${ }^{\ddagger} P<0.05$ vs. Val group; ${ }^{\S} P<0.05$ vs. VAml group.

Although we did not investigate cardiac sympathetic nerve activity, ${ }^{123}$ I-metaiodobenzylguanidine cardiac imaging previously suggested that cilnidipine suppresses cardiac sympathetic overactivity in individuals with hypertension, whereas amlodipine is largely without such an effect. ${ }^{32}$ These findings suggest that the differential effects of cilnidipine and amlodipine on cardiac sympathetic nerve activity are related to the difference in their cardioprotective effects. Our results indicate that, when combined with valsartan, cilnidipine also may suppress cardiac RAAS activity and exhibit synergistic or additive cardioprotective effects as a result of its inhibition of sympathetic neurotransmitter release mediated by $\mathrm{N}$-type calcium channel blockade.

Excess reactive oxygen species may also contribute to impairment of LV diastolic function through inhibition of $\mathrm{Ca}^{2+}$-handling proteins. ${ }^{33}$ Dihydropyridine derivatives act as lipophilic chain-breaking antioxidants. However, these drugs exhibit large differences in their lipophilicity, with cilnidipine being more lipophilic than amlodipine. ${ }^{34}$ Our present findings regarding cardiac oxidative stress are consistent with previous results showing that expression of the p2 $2^{\text {phox }}$ gene is regulated in a redox-sensitive manner in endothelial cells. ${ }^{35}$ The superior cardioprotective effects of valsartan plus cilnidipine in comparison with valsartan either alone or together with amlodipine may thus be attributable, at least in part, to the greater inhibition of cardiac oxidative stress by the former treatment. Loss of redox homeostasis results in activation of proinflammatory and profibrotic pathways in the heart, which in turn leads to diastolic dysfunction, likely as a result of increased LV diastolic stiffness. Collagen type I is thought to be a major determinant of myocardial stiffness. ${ }^{36}$ We have now shown that the upregulation of both collagen type I and type III genes apparent in the LVH group were attenuated to a greater extent in the VCil group than in the Val and VAml groups. Together, these data are consistent with previous results showing that the synthesis of collagen type I in cardiac fibroblasts is inhibited by antioxidants. ${ }^{37}$

Macrophages have been implicated in fibrosis associated with various pathological conditions. LV fibrosis, macrophage infiltration into the interstitial space of the myocardium and cardiac expression of the MCP-1 and osteopontin genes were attenuated to a greater extent in the VCil group than in the Val and VAml groups despite the similar lowering of SBP apparent in all three groups. The greater antiinflammatory and antifibrotic effects in the myocardium of the combination of valsartan plus cilnidipine are therefore likely, at least in part, independent of its antihypertensive effect. The upregulation of TGF- $\beta 1$ and connective tissue growth factor gene expression in the heart was also attenuated to a greater extent in the VCil group than in the Val and VAml groups. TGF- $\beta$-mediated diastolic dysfunction and fibrosis in pressure-overloaded hearts were previously associated with MCP-1-dependent macrophage accumulation, and these processes were suggested to be related to the RAAS. ${ }^{38,39}$

Given that cilnidipine exerts greater renoprotective effects than amlodipine when administered together with valsartan, ${ }^{17}$ combination therapy with cilnidipine and valsartan is a potential approach to the treatment of hypertensive patients. Several dihydropyridine CCBs have been found to possess mineralocorticoid receptor antagonist activity. ${ }^{40}$ Further studies are therefore warranted to elucidate the mechanisms underlying the superior effects of combination therapy with cilnidipine and valsartan. 
a

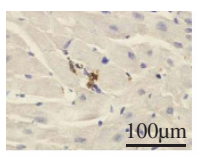

CONT

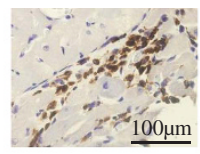

LVH

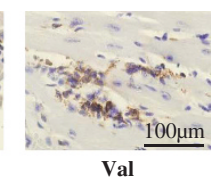

Val

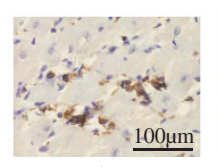

VAml

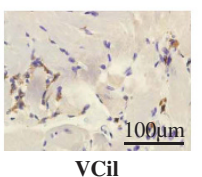

c

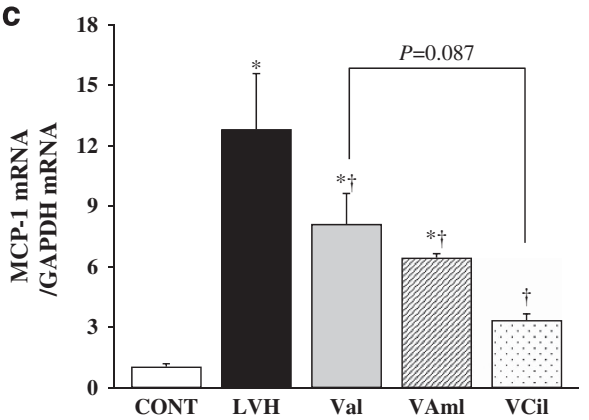

b

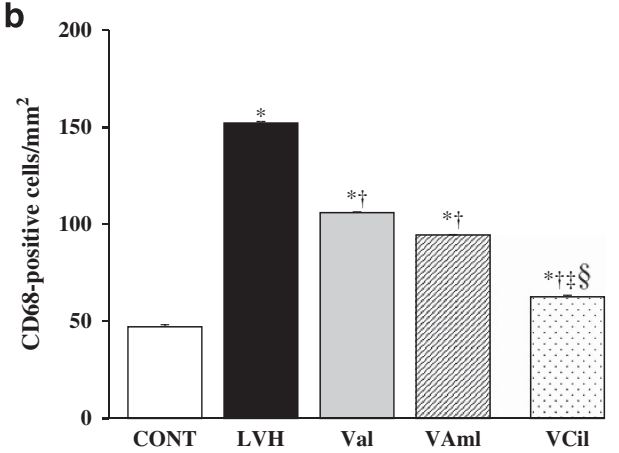

d

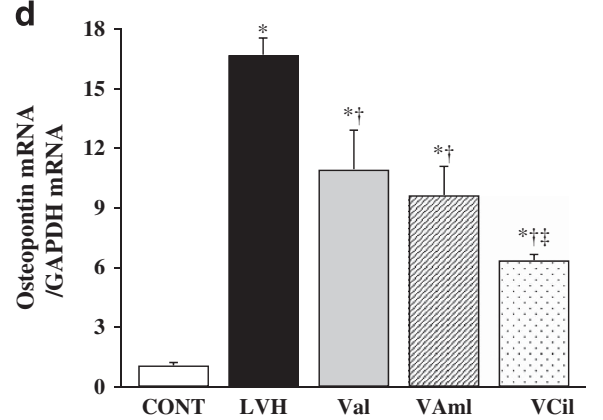

Figure 5 Macrophage infiltration as well as expression of MCP-1 and osteopontin genes in the left ventricle of rats in the five experimental groups at 11 weeks of age. (a) Immunohistochemical staining for the monocyte-macrophage marker CD68. Scale bars, $100 \mu \mathrm{m}$. (b) Density of CD68-positive cells in the LV myocardium as determined from sections similar to those in (a). (c, d) Quantitative RT-PCR analysis of MCP-1 and osteopontin mRNAs, respectively. The amount of each mRNA was normalized by that of GAPDH mRNA and then expressed relative to the mean value for the CONT group. Data in (b) through (d) are means \pm s.e.m. $\left(n=6,6,11,11\right.$ and 11 rats for the CONT, LVH, Val, VAml and VCil groups, respectively). ${ }^{*} P<0.05$ vs. CONT group; ${ }^{\dagger} P<0.05$ vs. LVH group; ${ }^{\ddagger} P<0.05$ vs. Val group; ${ }^{\S} P<0.05$ vs. VAml group.
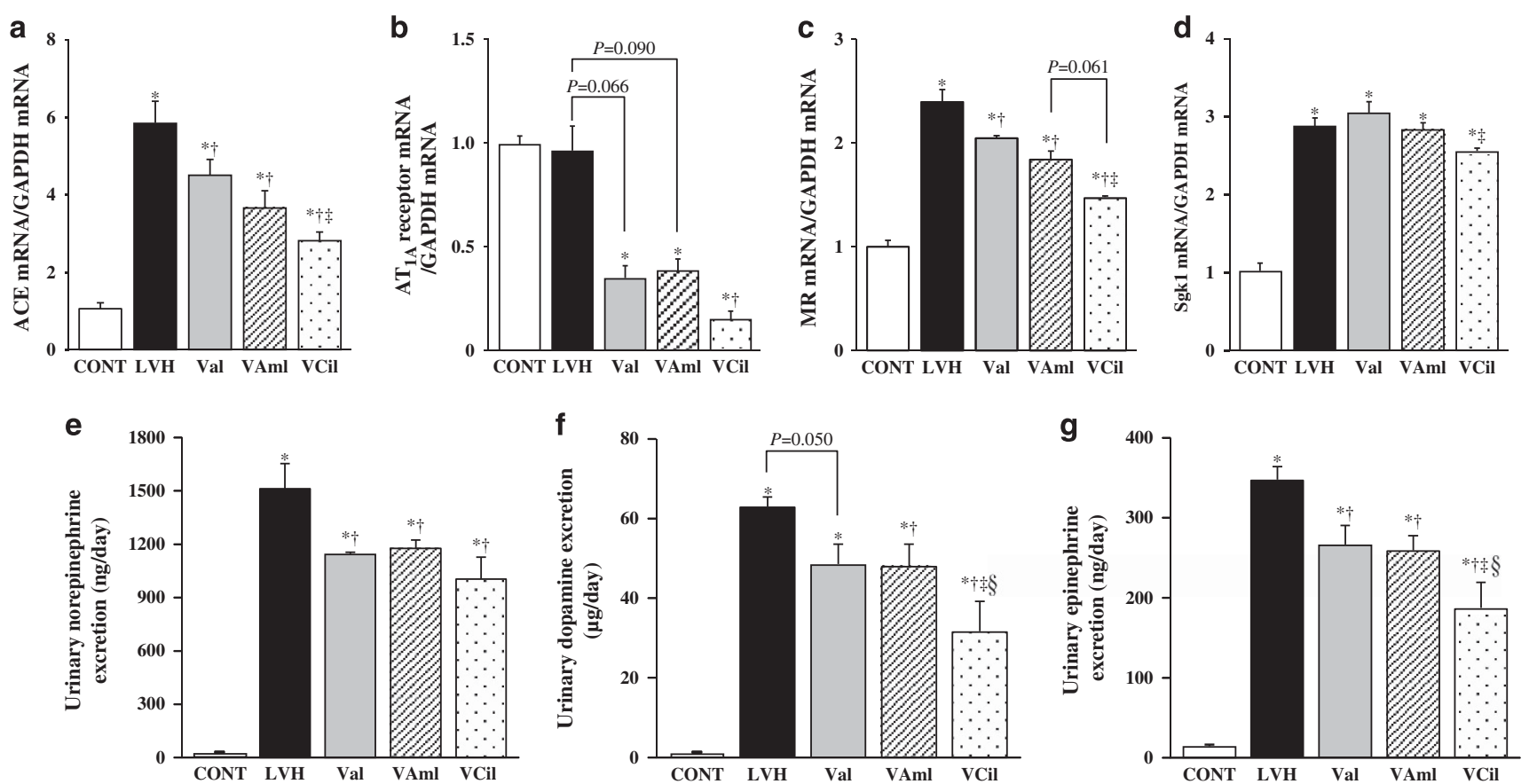

Figure 6 Expression of cardiac RAAS-related genes in the left ventricle as well as urinary excretion of catecholamines in rats of the five experimental groups at 11 weeks of age. (a-d) Quantitative RT-PCR analysis of angiotensin-converting enzyme (ACE), AT 1 A receptor, mineralocorticoid receptor (MR), and Sgk1 mRNAs, respectively. The abundance of each mRNA was normalized by that of GAPDH mRNA and then expressed relative to the mean value for the CONT group. (e-g) Urinary excretion of norepinephrine, dopamine and epinephrine, respectively. All data are means \pm s.e.m. $(n=6,6,11,11$ and 11 rats for the CONT, LVH, Val, VAml and VCil groups, respectively). ${ }^{*} P<0.05$ vs. CONT group; ${ }^{\dagger} P<0.05$ vs. LVH group; ${ }^{\ddagger} P<0.05$ vs. Val group; ${ }^{\S} P<0.05$ vs. VAml group. 
With regard to limitations of the present study, we collected a blood sample for measurements of renin activity and angiotensin II concentration in plasma under anesthesia by intraperitoneal injection of sodium pentobarbital because of our experiment protocols for multiple purposes. It is recommended to collect blood promptly by killing animals with rapid decapitation (unanesthetized) to prepare high quality blood samples, because anesthesia can influence sympathetic nerve activity to alter renin release from the kidney, leading to changes in plasma angiotensin II levels. Furthermore, plasma-derived or locally produced renin could be activated during the process for sample preparation, therefore increasing in vitro production of angiotensin II.

\section{CONCLUSIONS}

We have shown that valsartan monotherapy as well as combination therapy with valsartan and either amlodipine or cilnidipine similarly attenuated hypertension and LVH in DS hypertensive rats, and that the combination of valsartan and cilnidipine ameliorated LV concentricity, fibrosis and diastolic dysfunction to a greater extent than did the other two treatments. The superior cardioprotective action of valsartan plus cilnidipine compared with valsartan plus amlodipine is likely attributable, at least in part, to the greater antioxidant and antiinflammatory effects associated with both greater inhibition of cardiac RAAS gene expression and N-type calcium channel blockade.

\section{CONFLICT OF INTEREST}

The authors declare no conflict of interest.

\section{ACKNOWLEDGEMENTSACKNOWLEDGMENTS}

We thank Aya Wakamatsu, Yuuri Takeshita, Sae Ohura and Chieko Nakashima for technical assistance. This study was supported in part by unrestricted research grants from Ajinomoto Pharmaceuticals (Tokyo, Japan) and by Management Expenses Grants from the Japanese government to Nagoya University.

1 Thom T, Haase N, Rosamond W, Howard VJ, Rumsfeld J, Manolio T, Zheng ZJ, Flegal K, O'Donnell C, Kittner S, Lloyd-Jones D, Goff DC, Hong YL, Adams R, Friday G, Furie K, Gorelick P, Kissela B, Marler J, Meigs J, Roger V, Sidney S, Sorlie P, Steinberger J, Wasserthiel-Smoller S, Wilson M, Wolf P, American Heart Association Statistics Committee and Stroke Statistics Subcommittee. Heart disease and stroke statistics2006 update-A report from the American Heart Association Statistics Committee and Stroke Statistics Subcommittee. Circulation 2006; 113: E85-E151.

2 Rosamond W, Flegal K, Friday G, Furie K, Go A, Greenlund K, Haase N, Ho M, Howard V, Kissela B, Kittner S, Lloyd-Jones D, McDermott M, Meigs J, Moy C, Nichol G O'Donnell CJ, Roger V, Rumsfeld J, Sorlie P, Steinberger J, Thom aT, WasserthielSmoller S, Hong Y, American Heart Association Statistics Committee and Strok Statistics Subcommittee. Heart disease and stroke statistics-2007 update-A report from the American Heart Association Statistics Committee and Stroke Statistics Subcommittee. Circulation 2007; 115: E69-E171.

3 Ogihara T, Kikuchi K, Matsuoka H, Fujita T, Higaki J, Horiuchi M, Imai Y, Imaizumi T, Ito S, Iwao H, Kario K, Kawano Y, Kim-Mitsuyama S, Kimura G, Matsubara H, Matsuura H, Naruse M, Saito I, Shimada K, Shimamoto K, Suzuki H, Takishita S, Tanahashi N, Tsuchihashi T, Uchiyama M, Ueda S, Ueshima H, Umemura S, Ishimitsu T, Rakugi H, Japanese Society of Hypertension Committee. The Japanese Society of Hypertension guidelines for the management of hypertension (JSH 2009). Hypertens Res 2009 32: 3-107.

4 Oparil S, Weber M. Angiotensin receptor blocker and dihydropyridine calcium channel blocker combinations: an emerging strategy in hypertension therapy. Postgrad Med 2009; 121: 25-39.

5 Hayashi K, Ozawa Y, Fujiwara K, Wakino S, Kumagai H, Saruta T. Role of actions of calcium antagonists on efferent arterioles - with special references to glomerular hypertension. Am J Nephrol 2003; 23: 229-244.

6 Fujii S, Kameyama K, Hosono M, Hayashi Y, Kitamura K. Effect of cilnidipine, a novel dihydropyridine $\mathrm{Ca}^{2+}$-channel antagonist, on $\mathrm{N}$-type $\mathrm{Ca}^{2+}$ channel in rat dorsal root ganglion neurons. J Pharmacol Exp Ther 1997; 280: 1184-1191.

7 Uneyama H, Uchida H, Konda T, Yoshimoto R, Akaike N. Selectivity of dihydropyridines for cardiac L-type and sympathetic N-type $\mathrm{Ca}^{2+}$ channels. Eur J Pharmacol 1999; 373. 93-100.
8 Hirning LD, Fox AP, McCleskey EW, Olivera BM, Thayer SA, Miller RJ, Tsien RW. Dominant role of $\mathrm{N}$-type $\mathrm{Ca}^{2+}$ channels in evoked release of norepinephrine from sympathetic neurons. Science 1988; 239: 57-61.

9 Shiga T, Yamada Y, Matsuda N, Tanaka T, Urae A, Hashiguchi M, Hagiwara N, Kasanuki $\mathrm{H}$. Influence of cilnidipine or nisoldipine on sympathetic activity in healthy male subjects. Heart Vessels 2007; 22: 404-409.

10 Nagahama S, Norimatsu T, Maki T, Yasuda M, Tanaka S. The effect of combination therapy with an L/N-Type $\mathrm{Ca}^{2+}$ channel blocker, cilnidipine, and an angiotensin II receptor blocker on the blood pressure and heart rate in Japanese hypertensive patients: an observational study conducted in Japan. Hypertens Res 2007; 30: 815-822.

11 Zhou XY, Ono H, Ono Y, Frohlich ED. N- and L-type calcium channel antagonist improves glomerular dynamics, reverses severe nephrosclerosis, and inhibits apoptosis and proliferation in an I-NAME/SHR model. J Hypertens 2002; 20: 993-1000.

12 Kojima S, Shida M, Yokoyama H. Comparison between cilnidipine and amlodipine besilate with respect to proteinuria in hypertensive patients with renal diseases. Hypertens Res 2004; 27: 379-385.

13 Varagic J, Susic D, Frohlich ED. Cilnidipine improves spontaneously hypertensive rat coronary hemodynamics without altering cardiovascular mass and collagen. J Hypertens 2002; 20: 317-322.

14 Nagai H, Minatoguchi S, Chen XH, Wang NY, Arai M, Uno Y, Lu CJ, Misao Y, Onogi H, Kobayashi H, Takemura G, Maruyama R, Fujiwara T, Fujiwara H. Cilnidipine, an N+Ltype dihydropyridine $\mathrm{Ca}$ channel blocker, suppresses the occurrence of ischemia/ reperfusion arrhythmia in a rabbit model of myocardial infarction. Hypertens Res 2005; 28: 361-368.

15 Takatsu M, Hattori T, Murase T, Ohtake M, Kato M, Nashima K, Nakashima C, Takahashi K, Ito H, Niinuma K, Aritomi S, Murohara T, Nagata K. Comparison of the effects of cilnidipine and amlodipine on cardiac remodeling and diastolic dysfunction in Dahl salt-sensitive rats. J Hypertens 2012; 30: 1845-1855.

16 Fujita T, Ando K, Nishimura H, Ideura T, Yasuda G, Isshiki M, Takahashi K. Antiproteinuric effect of the calcium channel blocker cilnidipine added to reninangiotensin inhibition in hypertensive patients with chronic renal disease. Kidney Int 2007: 72: 1543-1549.

17 Aritomi S, Koganei H, Wagatsuma H, Mitsui A, Ogawa T, Nitta K, Konda T. The N-type and L-type calcium channel blocker cilnidipine suppresses renal injury in Dahl rats fed a high-salt diet. Heart Vessels 2010; 25: 549-555.

18 Kato MF, Shibata R, Obata K, Miyachi M, Yazawa H, Tsuboi K, Yamada T, Nishizawa T, Noda A, Cheng XW, Murate T, Koike Y, Murohara T, Yokota M, Nagata K. Pioglitazon attenuates cardiac hypertrophy in rats with salt-sensitive hypertension: role of activation of AMP-activated protein kinase and inhibition of Akt. J Hypertens 2008; 26: $1669-1676$

19 Nagata K, Iwase M, Sobue T, Yokota M. Differential effects of dobutamine and a phosphodiesterase inhibitor on early diastolic filling in patients with congestive heart failure. J Am Coll Cardiol 1995; 25: 295-304

20 Murase T, Hattori T, Ohtake M, Abe M, Amakusa Y, Takatsu M, Murohara T, Nagata K. Cardiac remodeling and diastolic dysfunction in DahIS.Z-Lepra/Leprfa rats: a new animal model of metabolic syndrome. Hypertens Res 2012; 35: 186-193.

21 Wang CT, Navar LG, Mitchell KD. Proximal tubular fluid angiotensin II levels in angiotensin II-induced hypertensive rats. J Hypertens 2003; 21: 353-360.

22 Ito K, Hirooka Y, Sunagawa K. Acquisition of brain Na sensitivity contributes to salt-induced sympathoexcitation and cardiac dysfunction in mice with pressure overload. Circ Res 2009; 104: 1004-U1200.

23 Miyata K, Rahman M, Shokoji T, Nagai Y, Zhang GX, Sun GP, Kimura S, Yukimura T, Kiyomoto $\mathrm{H}$, Kohno M, Abe $\mathrm{Y}$, Nishiyama A. Aldosterone stimulates reactive oxygen species production through activation of NADPH oxidase in rat mesangial cells. J Am Soc Nephrol 2005; 16: 2906-2912.

24 Sauzeau V, Jerkic M, Lopez-Novoa JM, Bustelo XR. Loss of Vav2 proto-oncogene causes tachycardia and cardiovascular disease in mice. Mol Biol Cell 2007; 18 943-952.

25 Sauzeau V, Sevilla MA, Rivas-Elena JV, de Alava E, Montero MJ, Lopez-Novoa JM, Bustelo XR. Vav3 proto-oncogene deficiency leads to sympathetic hyperactivity and cardiovascular dysfunction. Nat Med 2006; 12: 841-845.

26 Julius S. Sympathetic hyperactivity and coronary risk in hypertension. Hypertension 1993; 21: 886-893.

27 Mark AL. Sympathetic dysregulation in heart failure: mechanisms and therapy. Clin Cardiol 1995; 18: 13-18.

28 Minami J, Kawano Y, Makino Y, Matsuoka H, Takishita S. Effects of cilnidipine, a nove dihydropyridine calcium antagonist, on autonomic function, ambulatory blood pressure and heart rate in patients with essential hypertension. Br J Clin Pharmacol 2000; 50 615-620.

29 Konoshita T, Makino Y, Kimura T, Fujii M, Wakahara S, Arakawa K, Inoki I, Nakamura H, Miyamori IGenomic Disease Outcome Consortium Study I. A new-generation N/L-type calcium channel blocker leads to less activation of the renin-angiotensin system compared with conventional L type calcium channel blocker. J Hypertens 2010; 28 2156-2160.

30 Manabe I, Shindo T, Nagai R. Gene expression in fibroblasts and fibrosis-Involvement in cardiac hypertrophy. Circ Res 2002; 91: 1103-1113.

31 Zoccali C, Mallamaci F, Tripepi G, Parlongo S, Cutrupi S, Benedetto FA, Cataliotti A, Malatino LS, Investigators C. Norepinephrine and concentric hypertrophy in patients with end-stage renal disease. Hypertension 2002; 40: 41-46.

32 Sakata K, Shirotani M, Yoshida H, Nawada R, Obayashi K, Togi K, Miho N. Effects of amlodipine and cilnidipine on cardiac sympathetic nervous system and neurohormonal status in essential hypertension. Hypertension 1999; 33: 1447-1452. 
33 Adachi T, Weisbrod RM, Pimentel DR, Ying J, Sharov VS, Schoneich C, Cohen RA. S-glutathiolation by peroxynitrite activates SERCA during arterial relaxation by nitric oxide. Nat Med 2004; 10: 1200-1207.

34 Uesawa Y, Mohri K. Relationship between lipophilicities of 1,4-dihydropyridine derivatives and pharmacokinetic interaction strengths with grapefruit juice. J Pharm Soc Japan 2008; 128: 117-122.

35 Djordjevic T, Pogrebniak A, BelAiba RS, Bonello S, Wotzlaw C, Acker H, Hess J, Gorlach A. The expression of the NADPH oxidase subunit $\mathrm{p} 22^{\text {phox }}$ is regulated by a redoxsensitive pathway in endothelial cells. Free Radic Biol Med 2005; 38: 616-630.

36 Heeneman S, Cleutjens JP, Faber BC, Creemers EE, van Suylen RJ, Lutgens E, Cleutjens KB, Daemen MJ. The dynamic extracellular matrix: intervention strategies during heart failure and atherosclerosis. J Pathol 2003; 200: 516-525.
37 Chen K, Chen JW, Li DY, Zhang XJ, Mehta JL. Angiotensin II regulation of collagen type I expression in cardiac fibroblasts-modulation by PPAR- $\gamma$ ligand pioglitazone. Hypertension 2004; 44: 655-661.

38 Kuwahara F, Kai H, Tokuda K, Takeya M, Takeshita A, Egashira K, Imaizumi T. Hypertensive myocardial fibrosis and diastolic dysfunction-another model of inflammation? Hypertension 2004; 43: 739-745.

39 Tokuda K, Kai H, Kuwahara F, Yasukawa H, Tahara N, Kudo H, Takemiya K, Koga M, Yamamoto T, Imaizumi T. Pressure-independent effects of angiotensin II on hypertensive myocardial fibrosis. Hypertension 2004; 43: 499-503.

40 Dietz JD, Du S, Bolten CW, Payne MA, Xia C, Blinn JR, Funder JW, Hu X. A number of marketed dihydropyridine calcium channel blockers have mineralocorticoid receptor antagonist activity. Hypertension 2008; 51: 742-748. 